\title{
Wellen's Syndrome
}

\section{Željko Delići ${ }^{1}$, Slavica Ćirković2 ${ }^{\text {, Danijela Vuković }}{ }^{1}$, Milenko Čanković3 ${ }^{3}$, Dragan Debeljački ${ }^{3}$, Mila Kovačević3}

${ }^{1}$ General Hospital Vrbas, ${ }^{2}$ General Hospital Požarevac, ${ }^{3}$ Institute for cardiovascular diseases Vojvodina, Sremska Kamenica, Serbia

Abstract The pattern of clinical findings and electrocardiography ( ECG ) changes known as Wellens' syndrome is associated with significant stenosis of the proximal left anterior descending coronary artery ( LAD ). ECG changes are classified into two types : Type A (with biphasic T waves) or Type B (deep negative T waves, especially in leads V2and V3).

We present a case of Wellen's syndrome in which the ECG pattern changed from type B into type A during the hospitalization, and in which the coronary lesion is in the middle, rather than in the proximal part of LAD.

Key words Wellens' syndrome, electrocardiography, left anterior descending coronary artery

\section{Introduction}

n 1982 Wellens et al. first published the clinical and ECG criteria of a subgroup of patients with myocardial ischemia that later came to be known as Wellen's syndrome. ${ }^{1}$ The presence of Wellen's syndrome has significant diagnostic and prognostic value. Wellen's sign has a positive predictive value of $86 \%$. In the original Wellens' group, $75 \%$ of those with typical manifestations of the syndrome had a anterior myocardial infarction. Although, not one of the indication for $\mathrm{pPCl}$, each patients with the typical ECG finding, should be considered for coronary angiography and reperfusion. ${ }^{2-4}$

\section{Case Report}

72 years, male patient, F. V. was admitted to Coronary Care Unit because of recurrent chest pain from the previous day, duration of ten minutes. They were presented with the initial pain in the arms, shoulders with spread in the middle of the chest ("centripetal projection") unlike more frequent anginal pain presented by the "centrifugal pain projections". There was no vegetative symptoms. At the time of admission, the patient was asymptomatic, cardiopulmonary and metabolic compensated, in sinus rhythm,BP 120/70 mmHg. Physical examination was unremarkable. Among the risk factors for coronary artery disease, several years of smoking experience has been recorded, as well as information of Sudden cardia death of his father at the age of 40 . The patient have similar pains in the past, but not in this frequency and intensity, and tied them to the existence of chronic degenerative changes in the cervicale spine.

In the laboratory findings, cardiac enzymes $\mathrm{CK}$ and CKMB were in the reference value, with Troponin I at the upper reference value of 0.11 . The first control sample of CK and CKMB show no characteristic increase, and Troponin value was 0.22 . Echocardiography not find
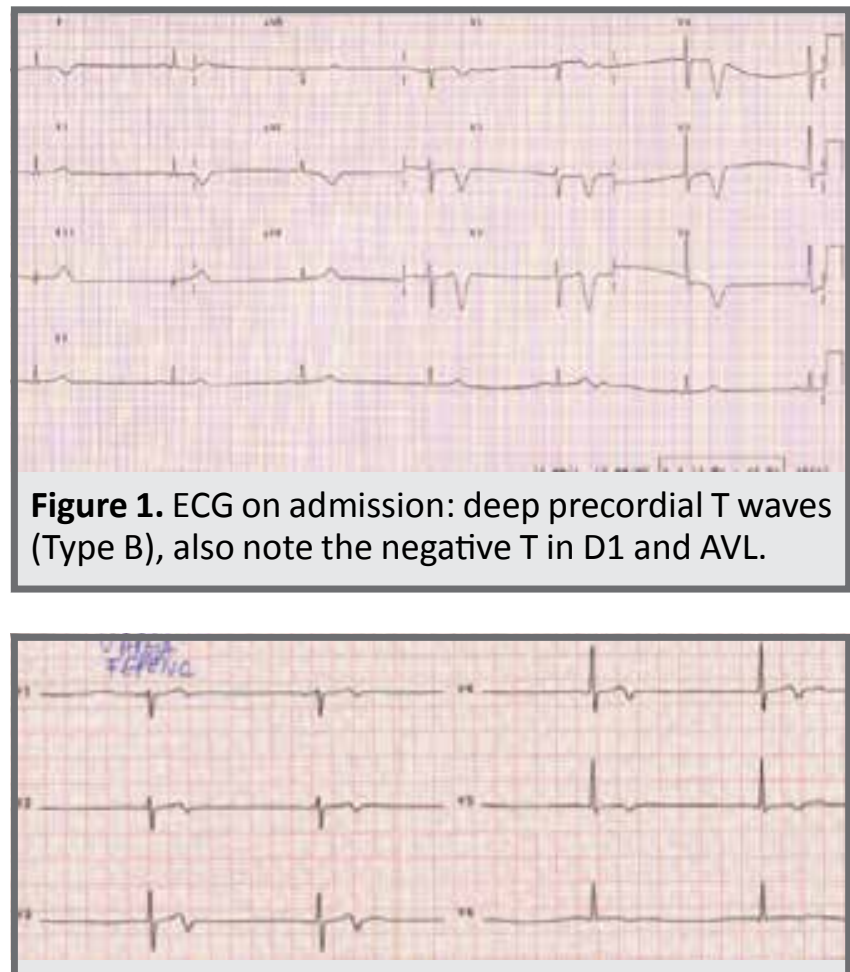

Figure 2. ECG with characteristic biphasic T wave V2, V3 (V4). (Type A) $5^{\text {th }}$ day of hospitalization

left ventricle segment kinetics disorders, as well as no signs of hypertrophic myocardium. Further controlled alterations of cardiac enzymes levels (without further monitoring TNIU) remains the reference interval. On the $5^{\text {th }}$ day of hospitalization, electrocardiographic findings changes with transformation of deep negative $\mathrm{T}$ in V2 and V3 in a clear biphasic T waves in the same leads.

It is important to remember, that most of the time, these typical ECG signs may be present only during periods without pain, which was confirmed in our case 


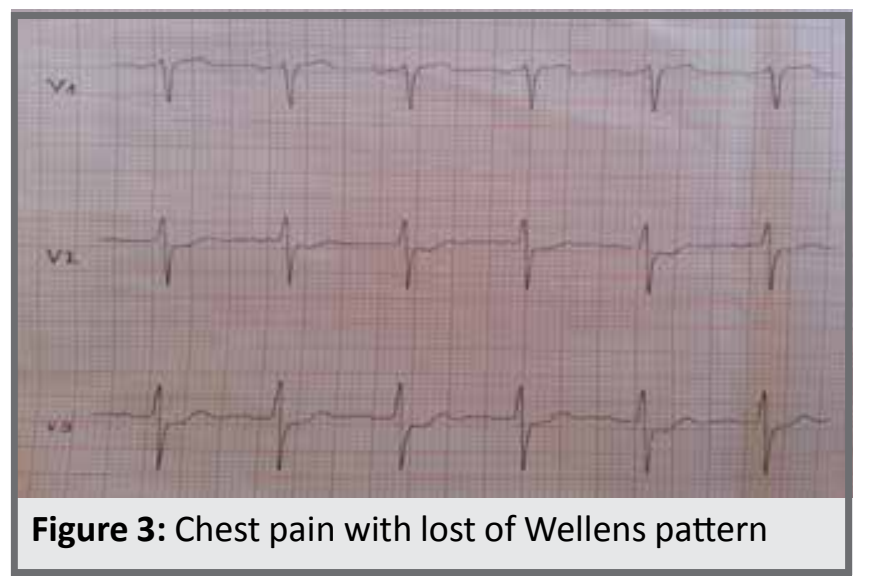

too. $T$ waves changes are usually present in the leads V2 and V3, though, in some cases, leads V1 and V4 can be also change. In the original Zwaan and Wellens study is recorded, that approximately $2 / 3$ of the respondents have a change in lead V1 and 3/4 of them includes change in lead V4. Our patient gets chest pain during 8 th $^{\text {th }}$ day of hospitalization with hypertensive reaction / BP 180/100 mmHg/, and his ECG lost Wellens sign. After spontaneous loosing of pain, ECG takes Wellens pattern again .TNIU was negative $(<0.01)$. Although, the mechanism remains unclear, its taken, that $\mathrm{T}$ waves changings, usually for a period without pain, is a result of easing spasm of LAD. ${ }^{5}$

Based on recurrent angina, finds of the characteristic ECG changes in the form of deep negative precordial $T$ waves, then the biphasic T V2, V3, the light increase of TNIU, preserved $R$ waves without $Q$, absence of the left ventricular segments kinetics disorders, we suspected on connection between changes with the proximal LAD occlusion. The case was presented to the interventional cardiologist, and the coronary angiography was performed. The patient was referred for coronary angiography . Coronary angiography: shows a significant narrowing of the medial part of LAD. After predilatation, 2 DES were placed in the medial part of the LAD. Flow through the LAD behind lesion and before the DES implantation was TIMI 1, and was clearly pointed on the "culprit" lesions.

After the procedure, the patient felt well,and he was discharged from the hospital $2^{\text {nd }}$ day.

\section{Discussion}

Wellens criterias were first described in 1982. by the Dutch De Zwaan and H.Wellens and are specific for the disease of LAD. It is necessery to recognize them, timely treatment to prevent the development of acute myocardial infarction and myocardial necrosis. ${ }^{1-3}$

\section{Simple criteria of Wellen's syndroma:}

- previous history of chest pain

- low or slightly elevated cardiac enzymes

- presence of precordial $R$ and absence of pathologi-

cal Q-wave

- small or insignificant ST elevation

- biphasic T in V2, V3 (type A)

- deep negative T V2, V3 (type B) [1]
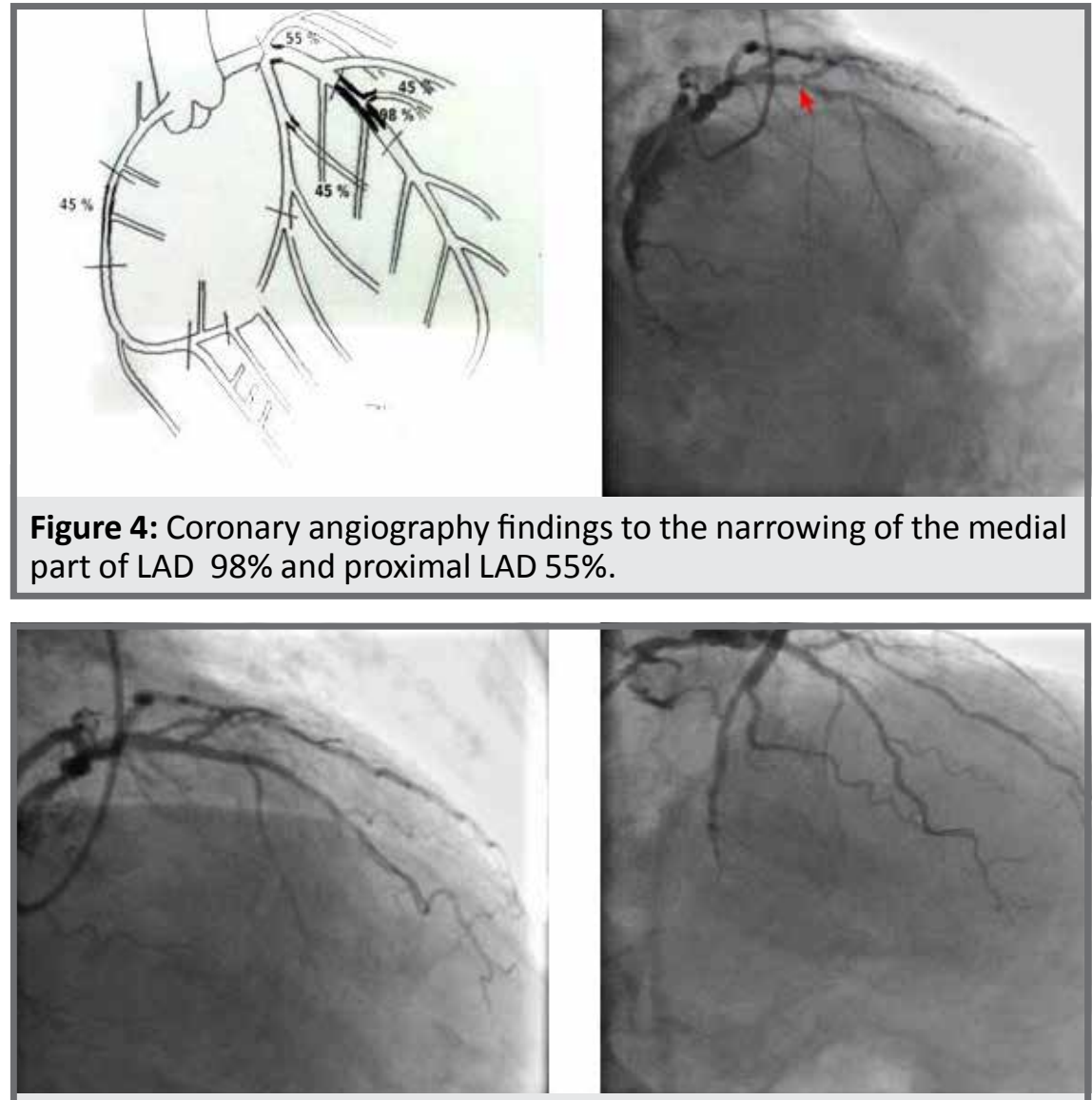

Figure 5. RAO i PA cranial after intervention. 
In $75 \%$ of cases, $\mathrm{T}$ waves are deep and inverted, whereas in $25 \%$ of cases, there are only biphasic T waves. Another important criteria is presence of precordial $R$ and absence of pathological Q- wave .The origin of these ECG changes remains obscure, as they can persist for months, but it is most likely that they represent reperfusion. ${ }^{6-7}$ Medical therapy in most of the cases is insufficient to prevent development of large myocardial infarction and demands early recognition and coronary percutaneous intervention. ${ }^{8}$

The sign can be seen as a rare presentation of :

- Takotsubo stress cardiomyopathy

- Cocaine-induced vasospasm

$-Q$ and non $Q$ myocardial infarction

- Myocarditis

- Pulmonary embolism

- CV Stroke

- WPW syndrome

- in the presence of left ventricular hypertrophy ect.

Table 1. Differential diagnosis and diagnostic procedures

\begin{tabular}{|l|c|c|c|c|c|}
\hline disease & Lab & ECG & Echo & Cor & MSCT \\
\hline Takotsubo & $\boldsymbol{V}$ & $\boldsymbol{V}$ & $\boldsymbol{V}$ & $\boldsymbol{V}$ & \\
\hline Cocaine vasosp. & $\boldsymbol{V}$ & $\boldsymbol{V}$ & $\boldsymbol{V}$ & & \\
\hline Myocardial inf. & $\boldsymbol{V}$ & $\boldsymbol{V}$ & $\boldsymbol{V}$ & $\boldsymbol{V}$ & \\
\hline Myocarditis & $\boldsymbol{V}$ & $\boldsymbol{V}$ & $\boldsymbol{V}$ & & \\
\hline Pulmonary emb. & $\boldsymbol{V}$ & $\boldsymbol{V}$ & $\boldsymbol{V}$ & & $\boldsymbol{V}$ \\
\hline CV stroke & $\boldsymbol{V}$ & $\boldsymbol{V}$ & $\boldsymbol{V}$ & & $\boldsymbol{V}$ \\
\hline WPW & & $\boldsymbol{V}$ & & & \\
\hline HLV & & $\boldsymbol{V}$ & $\boldsymbol{V}$ & & \\
\hline
\end{tabular}

\section{Risk Factors}

Risk factors for Wellens syndrome are traditional risk factors of coronary artery diseases, such as hypertension, diabetes mellitus, dyslipidemia, obesity, smoking, family history of coronary artery disease or events. Syndrome can be found in any age group. ${ }^{9}$ The youngest case reported in a 22-year-old man. ${ }^{10}$

\section{Therapy}

All patients with this syndrome have to receive conventional treatment of ACS including ASA, NGL, BB blockers if needed.

\section{Prognosis}

Most patient are feel good after percutaneous intervention. $T$ wave changes after the intervention may be withdrawn, so the ECG /of our patients after $\mathrm{PCl}$ is following:

\section{Conclusion}

Wellens syndrome is an important diagnostic and prognostic parameter, and it presents critical coronary artery disease involving $L A D$, which can lead to extensive anterior AMI, left ventricular failure, malignant arrhyth-

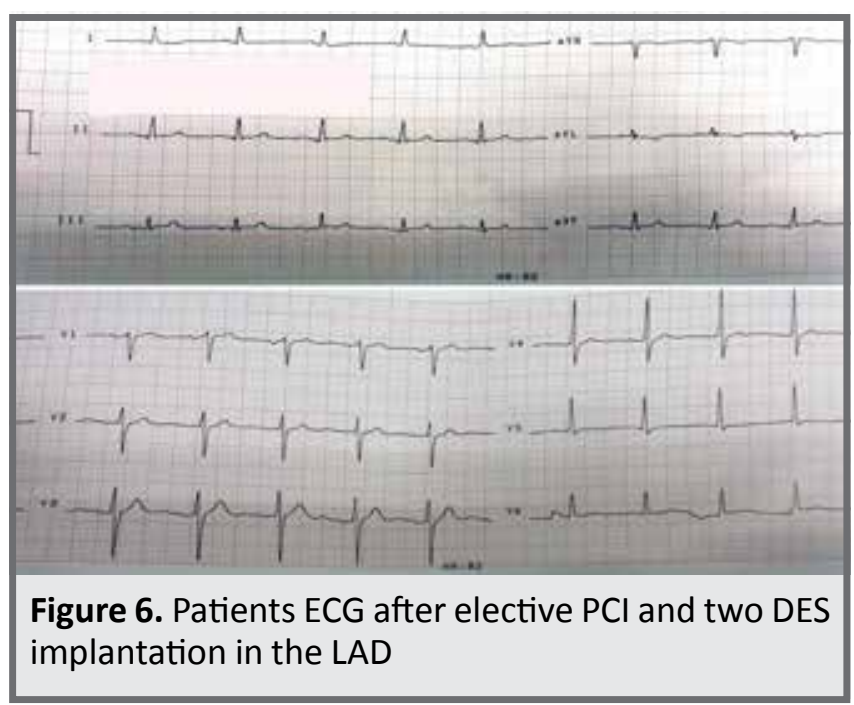

mia and death. Although patients may initially respond well to medical therapy, however, have a poor prognosis with conservative therapy, and all require invasive revascularization intervention. ${ }^{1}$ It is very important for clinicians to recognize the characteristic ECG patterns of Wellens syndroma.In our case $\mathrm{PCl}$ was perfomed before development of myocardial infarction.

\section{References}

1. Tatli E, Aktoz M, Wellens' syndrome and electrocardiography Cardiology Journal 2009, Vol. 16, No. 1, pp. 73-75.

2. Rhinehardt J, Brady WJ, Perron AD, Mattu A. Electrocardiographic manifestations of Wellens' syndrome. Am J Emerg Med 2002; 20(7): 638- 43.

3. De Zwaan C, Ba“r FW, Janssen JH, Cheriex EC, Dassen WR, Brugada $P$, et al. Angiographic and clinical characteristics of patients with unstable angina showing an ECG pattern indicating critical narrowing of the proximal LAD coronary artery. Am Heart J 1989; 117(3): 657- 65.

4. De Zwaan C, Ba“r FW, Wellens HJ. Characteristic electrocardiographic pattern indicating a critical stenosis high in left anterior descending coronary artery in patients admitted because of impending myocardial infarction. Am Heart J 1982; 103(4 Pt 2): 730 - 6.

5. Sheng FQ, He MR, Zhang ML, Shen GY. Wellens' syndrome caused by spasm of the proximal left anterior descending coronary artery. J Electrocardiol 2015; 48(3): 423 - 5.

6. Tandy TK, Bottomy DP, Lewis JG. Wellens' syndrome. Ann Emerg Med 1999;33:347- 51.

7. Rhinehard TJ, Brady WJ, Perron AD, et al. Electrocardiographic manifestations of Wellens' syndrome. Am J Emerg Med 2002;20:638-43.

8. Vanpee D, Courouble P, Marchandise B, Gillet JB. Wellens' syndrome. Ann Emerg Med 1999;34:684-5.

9. Khan B, Alexander J, Rathod KS, Farooqi F.Wellens' syndrome in a 24-year-old woman. BMJ Case Rep 2013; 2013: bcr2013 009323. doi: http://dx.doi. org/10.1136/bcr- 2013-009323

10. Wang JY, Chen H, Su X, Zhang ZP. Wellens' syndrome in a 22-year-old man. Am J Emerg Med 2016; 34: 937.e3-4. 